\title{
BMJ Open Detection of pulmonary nodules: a clinical study protocol to compare ultra- low dose chest CT and standard low- dose CT using ASIR-V
}

\author{
Marie Ludwig, ${ }^{1,2}$ Emilie Chipon, ${ }^{3,4}$ Julien Cohen, ${ }^{1,2}$ Emilie Reymond, ${ }^{1}$ \\ Maud Medici, ${ }^{3,4}$ Anthony Cole, ${ }^{1,2}$ Alexandre Moreau Gaudry, ${ }^{3,4}$ Gilbert Ferretti ${ }^{1,2}$
}

To cite: Ludwig M, Chipon E, Cohen J, et al. Detection of pulmonary nodules: a clinical study protocol to compare ultra-low dose chest CT and standard low-dose CT using ASIR-V. BMJ Open 2019;9:e025661. doi:10.1136/ bmjopen-2018-025661

- Prepublication history and additional material for this paper are available online. To view these files, please visit the journal online (http://dx.doi org/10.1136/bmjopen-2018025661).

Received 10 August 2018 Revised 20 June 2019 Accepted 21 June 2019

\section{Check for updates}

(c) Author(s) (or their employer(s)) 2019. Re-use permitted under CC BY-NC. No commercial re-use. See rights and permissions. Published by BMJ.

${ }^{1}$ Service de radiologie et imagerie médicale, pôle imagerie, Centre Hospitalier Universitaire Grenoble Alpes, Grenoble, France

${ }^{2}$ Faculte de Medecine, Universite Grenoble Alpes, La Tronche, France

${ }^{3}$ CIC 1406, INSERM, Grenoble,

France

${ }^{4}$ Pôle recherche, Centre Hospitalier Universitaire Grenoble Alpes, Grenoble, France

Correspondence to Dr Marie Ludwig; mludwig@ch-annecygenevois.fr and Mrs Emilie Chipon; echipon@chu-grenoble.fr

\section{ABSTRACT}

Introduction Lung cancer screening in individuals at risk has been recommended by various scientific institutions. One of the main concerns for CT screening is repeated radiation exposure, with the risk of inducing malignancies in healthy individuals. Therefore, lowering the radiation dose is one of the main objectives for radiologists. The aim of this study is to demonstrate that an ultra-low dose (ULD) chest CT protocol, using recently introduced hybrid iterative reconstruction (ASiR-V, GE medical Healthcare, Milwaukee, Wisconsin, USA), is as performant as a standard 'low dose' (LD) CT to detect non-calcified lung nodules $\geq 4 \mathrm{~mm}$.

Methods and analysis The total number of patients to include is 150 . Those are referred for non-enhanced chest CT for detection or follow-up of lung nodule and will undergo an additional unenhanced ULD CT acquisition, the dose of which is on average 10 times lower than the conventional LD acquisition. Total dose of the entire exam $(\mathrm{LD}+\mathrm{ULD})$ is lower than the French diagnostic reference level for a chest CT (6.65 millisievert). ULD CT images will be reconstructed with $50 \%$ and $100 \%$ ASiR-V and LD CT with $50 \%$. The three sets of images will be read in random order by two pair of radiologists, in a blind test, where patient identification and study outcomes are concealed. Detection rate (sensitivity) is the primary outcome. Secondary outcomes will include concordance of nodule characteristics; interobserver reproducibility; influence of subjects' characteristics, nodule location and nodule size; and concordance of emphysema, coronary calcifications evaluated by visual scoring and bronchial alterations between LD and ULD CT. In case of discordance, a third radiologist will arbitrate.

Ethics and dissemination The study was approved by the relevant ethical committee. Each study participant will sign an informed consent form.

Trial registration number NCT03305978; Pre-results.

\section{INTRODUCTION}

Lung cancer is the deadliest cancer in the world, ${ }^{1}$ mainly due to the fact that it is often diagnosed at advanced stages that are not surgically curable. The current challenge is therefore to detect lung cancer at early

\section{Strengths and limitations of this study}

- We will evaluate the sensitivity of an ultra-low dose (ULD) CT, delivering 10 times less radiation than conventional low-dose CT, to detect lung nodules, in a French population of 150 patients referred for lung nodule check-up or follow-up.

- We will use a recently introduced hybrid iterative reconstruction (ASiR-V), and different levels of ASiR-V will be assessed.

- Nodules characteristics will be analysed, in particular the diagnosis of intrapulmonary lymph node, which is a benign lesion.

- Patients with morbid obesity (body mass index $>35$ ) will not be included as image quality of ultra-low dose CT is not acceptable for those morphotypes.

- Readers will be aware of the type of CT acquisition (low dose and ULD) and reconstruction, because they are easily recognisable due to the different level of image noise.

asymptomatic stages. Risk factors such as smoking and occupational exposure (mainly asbestos, silica, arsenic, chromium, iron, coal and ionising radiation) are well known and enable to define the target population for such programmes.

The National Lung Screening Trial (NLST) was the first study to show that a low dose (LD) (average effective dose of $1.5 \mathrm{mSv}$ ) CT lung cancer screening reduced specific death by $20 \%(95 \%$ CI 6.8 to $26.7 ; \mathrm{p}=0.004)$ as compared with chest X-ray (CXR) screening (single-view posteroanterior) in actual or former smokers (>30 pack years) patients between 55 and 74 years old. ${ }^{2}$

Other lung cancer screening studies are still in progress in Europe, such as the NELSON study in Belgium and the Netherlands, the results of which are expected to be reported soon $^{3}$

However, the drawback of using LD CT at such doses $(<1.5$ millisievert $(\mathrm{mSv}))$ is that 


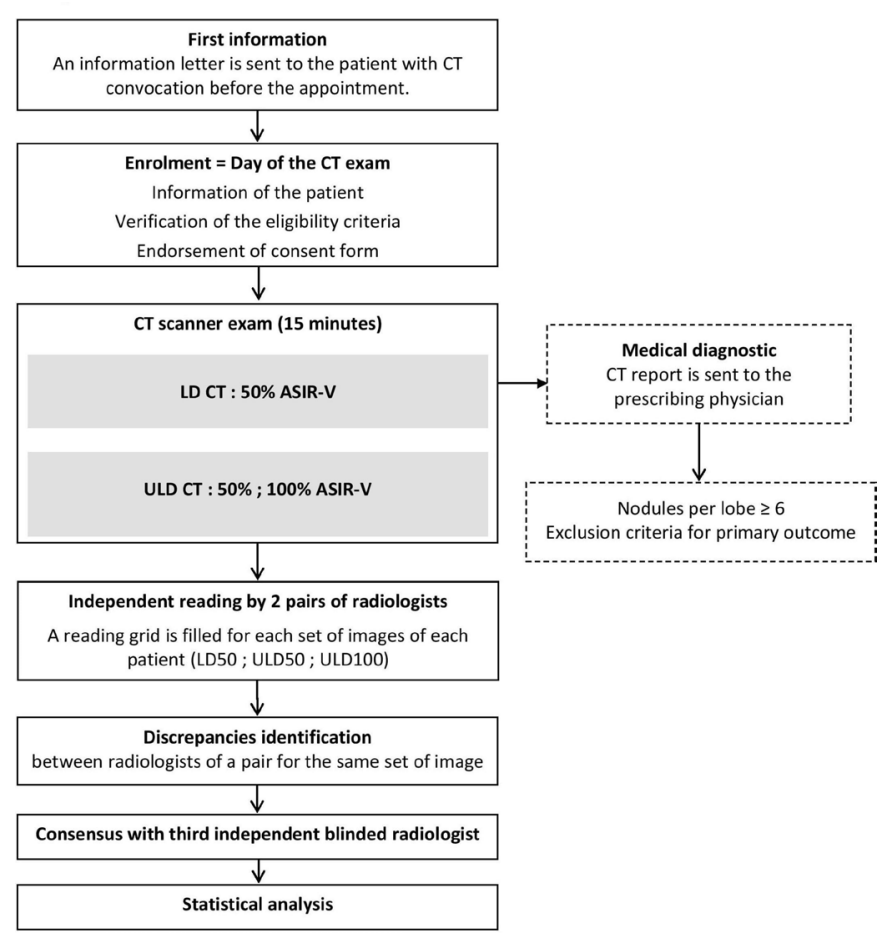

Figure 1 Study flow chart. ASiR-V , adaptive statistical iterative reconstruction-Véo (GE medical Healthcare, Milwaukee, Wisconsin, USA); LD, low dose; LD50, low dose CT with $50 \%$ ASiR-V reconstruction; ULD, ultralow dose; ULD50, ultra-low dose CT with 50\% ASiR-V reconstruction; ULD 100, ultra-low dose CT with 100\% ASIR-V reconstruction.

even though irradiating less than standard chest CT, the radiation exposure is still on average 10 times higher than a two views CXR and may be a risk for induced malignancies in itself. ${ }^{4}$

In this context, great efforts are currently being made by CT manufacturers to reduce the dose and maintain diagnostic quality. Technologies such as automated exposure control, lower tube current and iterative reconstruction ${ }^{5}$ were recently introduced, enabling further dose decrease for chest CTs, and the concept of "ultra-low dose (ULD) CT' (or submillisievert CT), which delivers a radiation dose approaching that of two CXR views at the cost of a slight deterioration of the image quality. ${ }^{6}$ Among these technological advances, the most significant is probably the new iterative reconstruction whether full iterative or hybrid. ${ }^{7-10}$

Promising results have been published for lung nodule detection with ULD CT. ${ }^{11-13}$ However, these studies were conducted on Asian populations, which may have different morphotypes compared with Caucasian populations.

Huber et al performed a phantom study comparing standard, LD and ULD CT for detection of pulmonary nodules. When compared with standard CT, the detection rate was $95.5 \%$ for LD CT $(1.76 \mathrm{mSv})$ and $93.3 \%$ for ULD CT $(0.13 \mathrm{mSv})$, increasing at $97.5 \%$ when adding computer-aided diagnosis and maximal intensity projection (MIP)..$^{14}$
Since we started to design our study protocol, Messerli et al published a study including 202 patients referred for any clinically indicated chest CT. A percentage of 91.2 nodules were detected using ULD CT $(0.13+/-0.01 \mathrm{mSv})$ as compared with LD CT $(1.8 \pm 0.7 \mathrm{mSv})$. Sensitivity was significantly higher for larger nodule diameter, lower body mass index (BMI) patients, lower image noise and for solid and calcified nodules. ${ }^{15}$

Neroladaki et al showed the same number of detected nodules between an ULD acquisition $(0.16 \pm 0.006 \mathrm{mSv})$ with iterative reconstruction and a standard dose filtered back projection acquisition $(11.2 \pm 2.7 \mathrm{mSv})$, and more nodules detected with model-based iterative reconstruction (MBIR) than adaptive statistical iterative reconstruction (ASIR) ${ }^{16}$ MBIR is known to better minimise image noise compared with ASIR: Ichikawa et al found a significantly lower image noise with $\mathrm{LD}(1.6 \pm 0.8 \mathrm{mSv})$ MBIR CT (11.6 \pm 1.0 Hounsfield units (HU)) than with LD ASIR CT $(21.1 \pm 2.6 \mathrm{HU}, \mathrm{p}<0.0005)$, a slightly better image quality score for decreased lung attenuation lesion, and no difference in image quality scores for consolidation or mass, ground-glass attenuation or reticular opacity with MBIR compared with ASIR LD CT. ${ }^{8}$ However, MBIR may slightly deteriorate lesion margin ${ }^{9}$ and significantly increases reconstruction time, taking more than $30 \mathrm{~min}$, when patients lie less than $10 \mathrm{~min}$ in the machine. ASiR-V is the latest generation of hybrid iterative reconstruction (GE Medical Healthcare, Milwaukee, Wisconsin, USA). It combines ASIR and MBIR and enables a better noise reduction than ASIR, with a processing time of only few minutes, suitable to a routine chest CT session. ${ }^{17}$

According to the as low as reasonably achievable (ALARA) principle, we hope to validate our ULD chest CT protocol $(<0.2 \mathrm{mSv})$, the dose of which is 10 times lower than a usual LD CT, as a sensitive tool to detect lung nodules. Thus, this ULD CT acquisition could be generalised for lung nodules detection and would consolidate the setup of lung cancer screening programmes. Also, this would allow the generalisation of ULD protocols, for radiation sensitive populations (children and young adults in particular).

\section{METHODS AND ANALYSIS}

\section{Study design and objectives}

The objectives of this study are to evaluate the performance of ULD CT for the detection of lung nodules, and the evaluation of nodule characteristics in comparison with LD CT. Furthermore, as smoking is a common risk factor, performance for the detection of cardiac and respiratory associated diseases (bronchial abnormalities, emphysema and coronary calcifications) is also evaluated.

An additional ULD CT is performed in patients referred for non-enhanced chest CT for lung nodules check-up or follow-up. The dose delivered with both acquisitions is still lower than the French diagnostic reference level $(6.65 \mathrm{mSv})$. We chose to only include nodules $\geq 4 \mathrm{~mm}$ as the incidence of cancer is very low below this threshold 
and is not currently considered as clinically significant. ${ }^{18}$ Nodules $<3 \mathrm{~mm}$ are considered as micronodules, and the recommendation from the Fleischner Society recommends that such nodules should not be measured, given inherent accuracy limitations and variability in determining whether the lesion is a solid, part-solid or groundglass nodule. ${ }^{19} \mathrm{~A} 4 \mathrm{~mm}$ threshold was also used for the NLST study. ${ }^{2}$ In addition, fully calcified nodules are excluded from the analysis because they are constantly benign and easily detected.

We will study nodule subtypes (solid, part-solid and pure ground-glass) and size. Furthermore, we will evaluate the performance of ULD CT to diagnose intrapulmonary lymph nodes, which are benign nodules not needing follow-up ${ }^{20}$ and were not analysed in previous ULD CT studies.

This trial sponsored by the Grenoble-Alpes University Hospital (CHUGA, France) is designed as a monocentric, prospective, non-randomised study in which the patient is his own control. All outcomes are evaluated by blinded double reading. Patient enrolment started in October 2017 and is expected to be completed in September 2018. Figure 1 summarises the process of inclusion, intervention and reading, described in detail below.

\section{Primary outcome}

Detection rate (sensitivity) of lung nodules in ULD chest CT using the conventional chest LD CT as gold standard.

\section{Secondary outcomes}

1. Diagnostic criteria: true positive (TP), false positive $(\mathrm{FP})$, true negative $(\mathrm{TN})$, false negative $(\mathrm{FN})$, positive predictive value (PPV), negative predictive value (NPV), specificity (Sp) of ULD CT.

2. Concordance of nodule's size, subtype and diagnosis of typical intrapulmonary lymph node among lung nodules between ULD and LD CT.

3. Interobserver reproducibility for size, subtype and diagnosis of lung nodules in ULD CT.

4. Influence of subjects characteristics (age, sex and $\mathrm{BMI}$ ), nodule location and nodule size on lung nodule detection with ULD CT.

5. Concordance of emphysema detection, type and distribution between ULD and LD CT.

6. Concordance of Weston score of coronary calcifications between ULD and LD CT.

7. Concordance of visual assessment of bronchial thickening, mucoid impaction or dilatation between ULD and LD CT.

\section{Eligibility criteria}

Inclusion criteria

- Aged 18 years or older.

- Referred for non-enhanced chest CT for the following indications:

- Lung nodule check-up or follow-up.

- Nodular abnormality on chest X-ray.
- Morphological assessment of chronic obstructive pulmonary disease or emphysema.

- Asbestos exposure.

- Assessment before lung radio frequency ablation.

- Assessment of disease extent of an extra thoracic cancer (in case of iodinated intravenous contrast agent contraindication)

- Check-up before extrathoracic transplantation (in case of iodinated intravenous contrast agent contraindication).

Exclusion criteria

- Inability to lie down and stay still during the examination.

- Inability to hold breath for more than $5 \mathrm{~s}$.

- Pneumonia in the last 3 months.

- BMI more than $35 \mathrm{~kg} / \mathrm{m}^{2}$.

- Pregnant or breastfeeding women.

\section{CT scan acquisitions and reconstructions}

The LD and ULD acquisitions are performed on the Revolution CT scanner (GE Medical Healthcare) equipped with the third generation ASiR-V iterative reconstruction. Acquisitions are performed successively in the same CT exam, in the supine position and at suspended full inspiration. Both acquisitions cover the same pulmonary fields from the apex to the costodiaphragmatic angle, determined on the scout views (two views).

The $\mathrm{LD}$ acquisition is the reference exam for the diagnosis of pulmonary nodules. The acquisition parameters are: spiral CT scanning; $120 \mathrm{kVp}$; automatic modulation of $3 \mathrm{D}$ radiation dose ('Smart $\mathrm{mA}$ ' + organ dose modulation) with lower bound $100 \mathrm{~mA}$, maximal bound $200 \mathrm{~mA}$ and noise index 10; rotation time: $0.35 \mathrm{~s}$; modulation $35-70$ $\mathrm{mAs}$; pitch $=0.992: 1$ and collimation: $80 \mathrm{~mm}$. The radiation dose, volume CT dose index (CTDIvol) and dose length product $(\mathrm{DLP})=\mathrm{CTDIvol} \times$ length of exposure) may vary depending on patient attenuation and length of the acquisition. The expected DLP is between 70 and 200 $\mathrm{mGy.cm}(0.98 \mathrm{mSv}$ to $2.8 \mathrm{mSv}$ ) (the effective dose is calculated by multiplying DLP by a thoracic conversion factor of $0.014^{21}$ ), for an average DLP of $100 \mathrm{mGy} . \mathrm{cm}$.

The ULD CT acquisition parameters are: spiral CT scanning: $120 \mathrm{kVp}$; fixed tube current of $10 \mathrm{~mA}$; rotation time: $0.35 \mathrm{~s}$; $3.5 \mathrm{mAs}$; pitch: $0.992: 1$; collimation: $80 \mathrm{~mm}$. These parameters are fixed for all patients. The CTDIvol is constant at $0.24 \mathrm{mGy}$. The DLP will depend only on the length of the acquired chest, different for each patient, expected around $10 \mathrm{mGy} . \mathrm{cm}(0.14 \mathrm{mSv})$. The modulation of the $\mathrm{mA}$ is deactivated to allow a very low tube current and therefore an ULD acquisition. The ULD acquisition increases the exam time by up to $2 \mathrm{~min}$.

The reconstruction parameters are identical for both acquisitions: slice thickness: $1.25 \mathrm{~mm}$; standard filter and lung filter; contiguous $8 \mathrm{~mm}$ thickness MIP reconstruction and iterative reconstruction with different percentages. We use ASiR-V in our study, which is the latest generation of iterative reconstruction techniques. It blends hybrid iterative reconstruction and standard filtered 
back projection. The percentage of ASiR-V represents the amount of iterative reconstruction from $0 \%$ (filtered back projection only) to $100 \%$ iterative reconstruction, which modifies image noise and texture. When designing our study, ASiR-V was not yet studied for chest CT. The CT vendor engineers suggested an empirical percentage between 40 up to $100 \%$, depending on radiologist practice and preferences. We decided to test percentages of iterative reconstruction of $50 \%$ and $100 \%$. The LD CT images are reconstructed with $50 \% \mathrm{ASiR}-\mathrm{V}$ (LD) and the ULD CT images with 50\% (ULD50) and 100\% (ULD100) ASiR-V.

The statistical analyses will be performed twice: with ULD50 and ULD100.

For every patient, CTDIvol and DLP are recorded. Effective dose and size-specific dose estimates will be then calculated. Concerning the additional radiation for included patients, our ULD CT protocol has an expected effective dose between $0.10 \mathrm{mSv}$ and $0.20 \mathrm{mSv}$, which is about 6-20 times lower than the LD protocol (which is the usual dose in our institution for this indication), similar to a two-view CXR and to 30 days of natural radiation. ${ }^{22}$ Moreover, total dose of the entire exam (around $1.1-3 \mathrm{mSv}$ ) is lower than French diagnostic reference level of $6.65 \mathrm{mSv}$.

\section{Recruitment and intervention}

Patients included in the study are those referred for a diagnostic chest CT without contrast media injection. On the day of the CT scan, a radiologist checks the eligibility criteria for the study and informs the patient who signs a participation consent form if he accepts to join the study. The radiologist then collects the following parameters: height, weight, history of oncology, cardiorespiratory pathology and exposure to smoking. The patient then undergoes the standard diagnostic LD CT acquisition followed by the ULD acquisition. If, however, the dose of the LD acquisition is greater than $6.65 \mathrm{mSv}$ (French diagnostic reference level), the ULD acquisition is not performed, and the patient is excluded from the data analysis. The patient's participation in the study is completed once he leaves the examination room.

The CT images of the LD acquisition are analysed by the radiologist who gives his medical report for the patient's medical management. If the number of nodules $\geq 4 \mathrm{~mm}$ identified on this acquisition is $\geq 6$ in one lung, the patient will be excluded from the data analysis because the analysis of the outcomes will be too complicated to implement.

\section{Patient and public involvement}

Patients or public were not directly involved in the development of the research question. However, lowering the radiation dose is a rising concern for the patients and for public health. Patients were also not directly involved in the design, the recruitment and the conduct of this study.

As a regular medical care, the report of the diagnostic LD CT is sent to the prescribing physician and to the patients at their request. According to French law, patients will be informed of the global results of the study at their request.

\section{Blind reading of outcomes}

For LD, ULD50 and ULD100 reconstructions, two radiologists will independently read all the radiological parameters. In order to limit the number of exams assessed by each reader, four radiologists split into two pairs will participate in the blind reading. Each pair of radiologists (one junior and one senior radiologist) reads the three sets of images for the same patient in a random order.

The term 'blind' means that radiologists have neither knowledge of the patient's identity nor access to the results of diagnostic reading. To avoid patient identification, CT acquisitions are anonymised by deleting in the Digital Imaging and Communications in Medicine (DICOM) fields: the name, age and date of birth of the patient; the date and time of the examination; and the name of the referring radiologist for the diagnosis. Each patient reconstruction is identified by a random number that differs for each of the two readers. Radiologists never read two series of the same patient consecutively.

Anonymised exams are periodically transmitted to a pair with at least $15 \mathrm{LD}, 15$ ULD50 and 15 ULD100 reconstructions. The three patient reconstructions are not necessarily given the same day to both radiologists. In addition, the order of presentation is not identical for the two radiologists.

The reading is performed on a diagnostic console (IMPAX software, 6.5.5.3502) (Agfa, Belgium) using Barco MDNC-3121monitors (Barco, Courtrai, Belgium) and includes mediastinal and parenchymal filter reconstructions for each acquisition. The radiologist is free to adapt the level and width of the window to its reading practice (initial parenchymal window defined by a width of $1500 \mathrm{UH}$ and a level of $-600 \mathrm{UH}$ ) and to perform multiplanar reconstructions in the different plans of space. The reading also includes the additional MIP reconstruction for each acquisition, in order to sensitise the detection of nodules ${ }^{23}$ (this type of reading from MIP series is performed in clinical routine).

Radiologists identify nodules of longer diameter $\geq 4 \mathrm{~mm}$ by locating them with the slice number and the lobe. It is known that each lung has three lobes (right upper lobe, middle lobe, right lower lobe, culmen, lingula and left lower lobe). Each radiologist completes a reading grid for each reconstruction with all detected nodule characteristics, evaluation of emphysema, coronary calcification and bronchial abnormalities.

The completed grids are given to a clinical research assistant for data entry and identification of discrepancies in identification of nodules between the two radiologists.

We consider that a nodule is the same between the two readers if:

- It is located in the same lobe.

- The slice number is identical at \pm 5 slices (a nodule will be visible on several successive slices).

- The longest diameter of nodule is the same at $\pm 2 \mathrm{~mm} .{ }^{24}$ 
If these criteria are not respected or if a radiologist identifies one or more nodules in addition to or less than the second radiologist, a consensus with a third CHUGA senior thoracic radiologist with 27 years of experience is obtained. This third radiologist is not part of the reading pairs. The consensus is made from anonymised reconstructions, and the reconstructions of the same patient are not processed successively.

Besides, for every reconstruction is recorded:

- Noise by measuring SD in a region of interest placed in the tracheal air above the carina.

- Shape of the trachea which indicates inspiration degree.

- Subjective image quality on a three-point scale.

\section{Data monitoring}

All data are monitored by Grenoble-Alpes University Hospital (trial sponsor), in order to verify that for every patient enrolled, there is a signed consent form and that the inclusion and exclusion criteria are respected. In addition, all data collected in the case report form of every enrolled patient are verified.

\section{Sample size}

With a $90 \%$ power, to have a sensitivity of detection of nodules with the ULD CT to $90 \%$ with a CI to $\pm 10 \%$, it would be necessary to analyse 124 nodules. According to a retrospective analysis of patients with indication of pulmonary nodule CT made at CHUGA, out of 420 patients per year with this indication, 210 present pulmonary nodules with a total of about 400 nodules. It should therefore include about 140 patients to have 124 nodules to be analysed. Considering a 5\% potential loss to follow-up or withdrawal of consent, the actual number of subjects to include is 147 in total. To this are added three potential patients who could be secondarily excluded from the study for a number of nodules $\geq 6$ in one of the lungs. The total number of patients to include is 150 . The sample size calculations were carried out using R software version 3.1.0 (library MKmisc, function power.diagnostic. test). ${ }^{25-27}$

\section{Statistical analysis}

In this non-randomised study where each patient is his own control, the threshold $0<0.05$ will be taken into account to define the significance of the statistical tests. Analyses will be carried out in accordance with good statistical analysis practices after freezing of the database and will be carried out with the software $\mathrm{R}$ (version $\geq 3.10 .0$ ).

The normality of the dependent quantitative parameters will be determined by the Shapiro-Wilks test or by graphical verification of the symmetry of the distribution. When the normality of the distribution of such a parameter has been demonstrated, it will be described by its mean and its SD. Otherwise it will be described by its median, the 25th and the 75th percentile. The qualitative parameters will be expressed in number and percentage.
For the main objective, the sensitivity of the ULD CT (compared with the LD CT) for the detection of nodules will be calculated and accompanied by a 95\% CI. For secondary objective 1 , the number of TP, FP, TN, FN, PPV, NPV and Sp of the ULD CT (compared with LD CT) will be calculated. For secondary objectives 2, 5, 6 and 7, the concordance of the qualitative variables will be evaluated using the kappa coefficient. The concordance of the quantitative variables will be evaluated using Lin's concordance coefficient. For each coefficient, the $95 \%$ CI will be given. For secondary objective 3 , interobserver reproducibility for qualitative variables will be evaluated using the kappa coefficient. It will be evaluated, for the quantitative variables, using the ICC (intraclass coefficient). For each coefficient, the $95 \%$ CI will be given. For secondary objective 4 , a logistic regression model will be implemented. The variable to be explained will be the result of detecting each nodule in ULD CT compared with the LD CT ( $0=$ good detection/1=bad detection). The explanatory variables will be the age, sex and BMI of the patient, the location (lobe) and the size of the nodule. The size of the nodule can be used as a qualitative variable $(<5 \mathrm{~mm}$, $5-10 \mathrm{~mm}$ and $>10 \mathrm{~mm}$ ).

An interim analysis including the analysis of the primary endpoint will be performed after inclusion of the first 50 patients. This interim analysis will aim to: decide whether to continue or stop the study for futility and readjust the number of patients if necessary (if the characteristics of the patients included do not correspond to those initially planned (too many patients without nodules $\geq 4 \mathrm{~mm}$ )). In order to maintain an overall threshold of $5 \%$ in the final analysis, the interim analysis will be carried out with a threshold of $0.1 \% .^{28}$ The results of the interim analysis will be taken into account by the steering committee to propose modifications to the analysis plan. For this interim analysis, data from the confrontation between the two radiologists will be used.

\section{Limitations}

First limitation of our protocol is that we do not have a true screening population because there is no organised lung cancer screening programme in our country yet. Therefore, our study population corresponds to patients routinely referred for lung nodule check-up or follow-up instead of a risk factor-based population.

Another limitation is that ULD CT is easily recognisable as the image noise is increased as compared with LD CT, as well as ULD 50 and ULD 100 are possible to distinguish for an experienced radiologist. As a consequence, readers were not blinded for these, but for patient name, sex, age, clinical status and CT report.

Recall bias is limited by a randomised order of presentation and cutting into several reading sessions.

Although we wanted to have a 'western population', we decided not to include obese patients with a BMI $>35$, because ULD CT are of poorer quality, due to the need of more radiation exposure to produce acceptable images. Vardhanabhuti et al recently found a loss of nodule 
detection with iterative reconstructed CT scanners at an effective dose of $0.14 \pm 0.01 \mathrm{mSv}$ for obese patients with $\mathrm{BMI}>38 .^{29}$

We decided to test percentage of $50 \%$ and $100 \%$ of ASiR-V. Tang et al tested ASiR-V from $10 \%$ to $100 \%$ in non-enhanced chest and showed ASiR-V has greater potential in reducing image noise and artefacts and maintaining image sharpness when compared with ASIR, and $60 \%$ ASIR-V had the highest image quality combining both the objective and subjective evaluation of images. ${ }^{30}$ This finding, although occurring after the design of our study, is close to our chosen $50 \%$ level of ASIR-V.

Finally, our study has been conceived before the recommendations of the EU Position statement published at the end of 2017. ${ }^{31}$ Therefore, we measured manually the nodules instead of using computerised volumetry.

\section{Ethics and dissemination}

This trial is registered on the ClinicalTrials.gov database (see online supplementary file 'trial registration data set'). The protocol version is $\mathrm{N}^{\circ} 1.0$ - Date: 4 May 2017. All patients sign a consent form before being enrolled in the trial, in accordance with the Declaration of Helsinki II. Once the statistical report is finalised, we plan to publish our results in an international scientific journal and present them in national and international congresses.

Acknowledgements The authors would like to thank Alexandre Rey and Pierre Pittet for their help to collect and prepare data, Tarek Ittobane for data monitoring, Dr A Jankowski for the inclusion of patients and Laura Cotarla for English proofreading.

Contributors $\mathrm{ML}$ is the corresponding author and contributed to the conception of the study, to the inclusion of patients, to the blind reading of outcomes and to the drafting of the manuscript. EC contributed to the conception of the study, to the drafting of the manuscript and is responsible for data management and its integrity. JC contributed to the conception of the study, to the inclusion of patients, to the blind reading of outcomes and to the revision of the manuscript. ER contributed to the inclusion of patients, to the blind reading of outcomes and to the revision of the manuscript. MM contributed to the design and application of statistical analysis and to the drafting of the manuscript. AC contributed to the blind reading of outcomes and to the revision of the manuscript. AMG contributed to the conception of the study and to the revision of the manuscript. GF is the principal investigator of the study and contributed to the conception of the work, to the inclusion of patients, to the blind consensus of outcomes and to the revision of the manuscript. All authors approved the final manuscript and agreed to be accountable for all aspects of the work.

Funding This trial is funded by the Delegation to Clinical Research and Innovation of Grenoble-Alpes University Hospital.

Competing interests None declared.

Patient consent for publication Not required.

Ethics approval This trial was approved by the relevant ethical committee (Comité de Protection des Personnes, CPP sud-est VI, France, 07/07/2017, CPP Reference: AU1342).

Provenance and peer review Not commissioned; externally peer reviewed.

Open access This is an open access article distributed in accordance with the Creative Commons Attribution Non Commercial (CC BY-NC 4.0) license, which permits others to distribute, remix, adapt, build upon this work non-commercially, and license their derivative works on different terms, provided the original work is properly cited, appropriate credit is given, any changes made indicated, and the use is non-commercial. See: http:// creativecommons.org/licenses/by-nc/4.0/.

\section{REFERENCES}

1. Globocan. Lung Cancer Estimated Incidence, Mortality and Prevalence Worldwide in 2012. http://globocan.iarc.fr/Pages/fact_ sheets_cancer.aspx.

2. Aberle DR, Adams AM, Berg CD, et al. Reduced lung-cancer mortality with low-dose computed tomographic screening. $N$ Engl $J$ Med 2011;365(5):395-409.

3. Yousaf-Khan $U$, van der Aalst $C$, de Jong PA, et al. Final screening round of the NELSON lung cancer screening trial: the effect of a 2.5year screening interval. Thorax 2017;72:48-56.

4. Committee to Assess Health Risks from Exposure to Low Levels of lonizing Radiation and National Research Council. Health risks from exposure to low levels of ionizing radiation: BEIR VII Phase 2. Washington, D.C: The National Academies Press, 2006.

5. Kubo T, Ohno Y, Seo JB, et al. Securing safe and informative thoracic CT examinations-Progress of radiation dose reduction techniques. Eur J Radiol 2017;86:313-9.

6. Kim Y, Kim YK, Lee BE, et al. Ultra-Low-Dose CT of the Thorax using iterative reconstruction: evaluation of image quality and radiation dose reduction. AJR Am J Roentgenol 2015;204:1197-202.

7. Vardhanabhuti V, Loader RJ, Mitchell GR, et al. Image quality assessment of standard- and low-dose chest CT using filtered back projection, adaptive statistical iterative reconstruction, and novel model-based iterative reconstruction algorithms. AJR Am J Roentgenol 2013;200(3):545-52.

8. Ichikawa Y, Kitagawa K, Nagasawa N, et al. CT of the chest with model-based, fully iterative reconstruction: comparison with adaptive statistical iterative reconstruction. BMC Med Imaging 2013;13:27.

9. Padole A, Singh S, Ackman JB, et al. Submillisievert chest CT with filtered back projection and iterative reconstruction techniques. AJR Am J Roentgenol 2014;203(4):772-81.

10. Montet X, Hachulla A-L, Neroladaki A, et al. Image quality of low mA CT pulmonary angiography reconstructed with model based iterative reconstruction versus standard CT pulmonary angiography reconstructed with filtered back projection: an equivalency trial. Eur Radiol 2015;25(6):1665-71.

11. Yoon HJ, Chung MJ, Hwang HS, et al. Adaptive statistical iterative reconstruction-applied ultra-low-dose CT with radiographycomparable radiation dose: usefulness for lung nodule detection. Korean J Radiol 2015;16(5):1132-41.

12. Sui $X$, Meinel FG, Song W, et al. Detection and size measurements of pulmonary nodules in ultra-low-dose CT with iterative reconstruction compared to low dose CT. Eur J Radiol 2016;85(3):564-70.

13. Nagatani $\mathrm{Y}$, Takahashi M, Murata K, et al. Lung nodule detection performance in five observers on computed tomography (CT) with adaptive iterative dose reduction using three-dimensional processing (AIDR 3D) in a Japanese multicenter study: Comparison between ultra-low-dose CT and low-dose CT by receiver-operating characteristic analysis. Eur J Radiol 2015;84:1401-12.

14. Huber A, Landau J, Ebner L, et al. Performance of ultralow-dose CT with iterative reconstruction in lung cancer screening: limiting radiation exposure to the equivalent of conventional chest $\mathrm{X}$-ray imaging. Eur Radiol 2016;26:3643-52.

15. Messerli M, Kluckert T, Knitel M, et al. Ultralow dose CT for pulmonary nodule detection with chest $x$-ray equivalent dose - a prospective intra-individual comparative study. Eur Radiol 2017;27:3290-9.

16. Neroladaki A, Botsikas D, Boudabbous S, et al. Computed tomography of the chest with model-based iterative reconstruction using a radiation exposure similar to chest $\mathrm{X}$-ray examination: preliminary observations. Eur Radiol 2013;23(2):360-6.

17. De Marco P, Origgi D. New adaptive statistical iterative reconstruction ASiR-V: Assessment of noise performance in comparison to ASiR. J App/ Clin Med Phys 2018;19:275-86.

18. Gierada DS, Pinsky P, Nath $\mathrm{H}$, et al. Projected outcomes using different nodule sizes to define a positive CT lung cancer screening examination. J Natl Cancer Inst 2014;106:dju284.

19. Bankier AA, MacMahon H, Goo JM, et al. Recommendations for measuring pulmonary nodules at CT: a statement from the Fleischner Society. Radiology 2017;285:584-600.

20. Ahn Ml, Gleeson TG, Chan IH, et-al ClH, et al. Perifissural nodules seen at CT screening for lung cancer. Radiology 2010;254:949-56.

21. Task Group on Control of Radiation Dose in Computed Tomography. Managing patient dose in computed tomography. A report of the International Commission on Radiological Protection. Ann ICRP 2000;30(4):7-45.

22. Exposition de la population française aux rayonnements ionisants liée aux actes de diagnostic médical en 2012. Rapport IRSN PRPHOM N²014-6.

23. Jankowski A, Martinelli T, Timsit JF, et al. Pulmonary nodule detection on MDCT images: evaluation of diagnostic performance using thin 
axial images, maximum intensity projections, and computer-assisted detection. Eur Radiol 2007;17(12):3148-56.

24. Revel MP, Bissery A, Bienvenu M, et al. Are two-dimensional CT measurements of small noncalcified pulmonary nodules reliable? Radiology 2004;231:453-8.

25. Flahault A, Cadilhac M, Thomas G. Sample size calculation should be performed for design accuracy in diagnostic test studies. $J$ Clin Epidemiol 2005;58:859-62.

26. Chu H, Cole SR. Sample size calculation using exact methods in diagnostic test studies. J Clin Epidemiol 2007;60:1201-2.

27. M.R. Ccyl. The saw-toothed behavior of power versus sample size and software solutions: single binomial proportion using exact methods. Am Stat 2002;56:149-55.
28. Schulz KF, Grimes DA. Multiplicity in randomised trials II: subgroup and interim analyses. Lancet 2005;365:1657-61.

29. Vardhanabhuti V, Pang CL, Tenant S, et al. Prospective intraindividual comparison of standard dose versus reduced-dose thoracic CT using hybrid and pure iterative reconstruction in a followup cohort of pulmonary nodules-Effect of detectability of pulmonary nodules with lowering dose based on nodule size, type and body mass index. Eur J Radiol 2017;91:130-41.

30. Tang H, Yu N, Jia Y, et al. Assessment of noise reduction potential and image quality improvement of a new generation adaptive statistical iterative reconstruction (ASIR-V) in chest CT. Br J Radiol 2018:91:20170521.

31. Oudkerk M, Devaraj A, Vliegenthart R, et al. European position statement on lung cancer screening. Lancet Oncol 2017;18:e754-66. 